EPJ Web of Conferences 49, 18029 (2013)

DOI: $10.1051 /$ epjconf/20134918029

(C) Owned by the authors, published by EDP Sciences, 2013

\title{
Search for the standard model Higgs boson decaying to tau pairs produced in association with a $\mathrm{W}$ or $\mathrm{Z}$ boson
}

\author{
Abdollah Mohammadi ${ }^{1 a}$ for the CMS Collaboration \\ ${ }^{1}$ Université Libre de Bruxelles(ULB) and Interuniversity Institute for High Energies (ULB-VUB), Belgium
}

\begin{abstract}
A search for the standard model Higgs boson decaying to tau pairs is presented using data collected in 2011 and 2012 with the CMS detector at the LHC. The topologies studied represent three or four lepton final states, where the Higgs boson is produced in association with the leptonic decay of a W or Z boson, respectively. The analyses use collision data samples corresponding to integrated luminosities of $5 \mathrm{fb}^{-1}$ collected at $\sqrt{s}=7$ $\mathrm{TeV}$ and $12 \mathrm{fb}^{-1}$ of $8 \mathrm{TeV}$. Upper limits between 2.7 and 5.5 times the standard model prediction are established on the product of Higgs boson cross section and tau pair decay branching fraction for Higgs masses between 110 and $145 \mathrm{GeV}$.
\end{abstract}

\section{Introduction}

A resonance consistent with a SM Higgs boson with a mass of about $125 \mathrm{GeV}$ has been observed with a significance of $5.0 \sigma(5.9 \sigma)$ at the CMS [1] (ATLAS [2]) experiments. At both experiments, the observed excess is driven by the $\mathrm{H} \rightarrow \gamma \gamma, \mathrm{H} \rightarrow \mathrm{ZZ}$ and $\mathrm{H} \rightarrow \mathrm{WW}$ decay modes. While the data are insufficient to exclude the presence of a SM Higgs boson decaying to tau pairs, no significant excess has been observed in any $\mathrm{H} \rightarrow \tau^{+} \tau^{-}$searches $[1,3]$. It is therefore critical to measure this new resonance in its decays to tau pairs, to determine if it is consistent with the SM Higgs boson or not.

In this document we present additional searches for the Higgs boson decaying to a tau pair, in two final state topologies, where the Higgs boson is produced in association with the leptonic decay of a $\mathrm{W}$ or $\mathrm{Z}$ boson[4]. While the decays to tau pairs are the dominant Higgs boson signal contribution, the final states used in this paper can additionally be produced by the decay of the Higgs boson into a pair of $\mathrm{W}$ bosons that both decay to leptons. Although the cross section production for the SM Higgs via associated production mechanism is an order of magnitude lower than that of the gluon-gluon fusion, the presence of isolated high momentum leptons originating from $\mathrm{W}$ and $\mathrm{Z}$ decays suppresses the backgrounds dramatically, making these channels viable for searches for the Higgs boson.

The searches use a data sample of proton-proton collisions from an integrated luminosity of $17 \mathrm{fb}^{-1}$ recorded by the Compact Muon Solenoid (CMS) [5] experiment at the LHC. The data are separated into two periods: $5 \mathrm{fb}^{-1}$ was collected in 2011 at $\sqrt{s}=7 \mathrm{TeV}$, and $12 \mathrm{fb}^{-1}$ was collected in 2012 at $\sqrt{s}=8 \mathrm{TeV}$. Throughout this document, the expression "light lepton," or symbol $\ell$, will refer

\footnotetext{
ae-mail: abdollah.mohammadi@cern.ch
}

to an electron or muon, the symbol $\tau_{h}$ to a hadronicallydecaying tau, and the symbol $L$ to an e, $\mu$, or $\tau_{h}$.

\section{WH Selection}

Events are selected online using the double muon or $\mu+\mathrm{e}$ trigger, in the $\mu \mu \tau$ and e $\mu \tau$ channels, respectively. The leading (subleading) light lepton candidate is required to have $p_{\mathrm{T}}>20 \mathrm{GeV}(10 \mathrm{GeV})$. The $\tau_{h}$ candidate is required to have $p_{\mathrm{T}}>20 \mathrm{GeV}$ and pass the tight muon rejection and MVA electron rejection [3]. The probability for a quark or gluon jet to pass the $\tau$ identification is 10 to 100 times greater than the probability for a jet to pass the electron or muon identification. To remove the large background from $\mathrm{Z} \rightarrow L L$ events with a quark or gluon jet misidentified as a $\tau$ candidate, the two light leptons $(\mathrm{e} \mu, \mu \mu)$ are required to have the same charge.

To suppress ZZ background events, events with additional identified electrons and muons are removed. Events containing jets with $p_{\mathrm{T}}>20 \mathrm{GeV}$ and $|\eta|<2.4$ that are identified as coming from b-quarks are discarded to suppress $t \bar{t}$ background events. The lepton candidates in reducible background events have a softer $p_{\mathrm{T}}$ spectrum than those coming from Higgs boson production. These backgrounds are reduced by requiring that the scalar sum of the $E_{\mathrm{T}}$ of the lepton candidates $\left(L_{T}\right)$ be greater than $80 \mathrm{GeV}$.

\section{ZH Selection}

The search for $\mathrm{ZH}$ production is performed in four-lepton events with a reconstructed pair of electrons or muons consistent with the decay of a $\mathrm{Z}$ boson, and a tau pair (Higgs boson) candidate. The dominant backgrounds are reducible $Z+2$ jet events, where the jets are misidentified as the Higgs boson candidate, and irreducible $\mathrm{ZZ}$ production. Four final states are considered for the Higgs boson 
candidate: $\mathrm{e} \mu, \mathrm{e} \tau_{h}, \mu \tau_{h}$, and $\tau_{h} \tau_{h}$. The events are selected online using either a double muon or a double electron trigger. The leading and sub-leading leptons associated to the $\mathrm{Z}$ boson are required to have $p_{\mathrm{T}}>20 \mathrm{GeV}$ and 10 $\mathrm{GeV}$, respectively, and be of opposite charge, and the total energy deposit in the isolation region $(I)$ must be less than $30 \%$ of the lepton $p_{\mathrm{T}}$. To reject background events with leptonic decays of $\mathrm{t} \overline{\mathrm{t}}$, the invariant mass of the $\mathrm{Z}$ candidate is required to satisfy $60<m_{\ell \ell}<120 \mathrm{GeV}$. Events containing jets with $p_{\mathrm{T}}>20 \mathrm{GeV}$ and $|\eta|<2.4$ that are identified as coming from b-quarks are discarded to further suppress $\mathrm{t} \overline{\mathrm{t}}$ background events. The leptons associated to the Higgs boson candidate are required to have opposite charge, $p_{\mathrm{T}}>10 \mathrm{GeV}$ if an electron or muon, and $p_{\mathrm{T}}>$ $20 \mathrm{GeV}$ if a $\tau_{h}$ candidate. The object isolation requirements depend on the subchannel, depending on the contributions from the reducible and irreducible background processes. To reduce the contribution from $\mathrm{Z}+$ jets backgrounds, muons are required to have $I / p_{\mathrm{T}}<0.15(0.30)$ in the $\ell \ell \mu \tau_{h}(\ell \ell \mathrm{e} \mu)$ channel, electrons are required to have $I / p_{\mathrm{T}}<0.10(0.30)$ in the $\ell \ell \mathrm{e} \tau_{h}(\ell \ell \mathrm{e} \mu)$ channel, and $\tau_{h}$ candidates are required to pass the tight (medium) combined isolation in the $\ell \ell \tau_{h} \tau_{h}\left(\ell \ell \mathrm{e} \tau_{h}, \ell \ell \mu \tau_{h}\right)$ channels.

\section{Background Estimation}

The reducible backgrounds have at least one non-prompt lepton and are estimated solely using data.

The misidentification probabilities as a function of candidate $p_{\mathrm{T}}$ for non-prompt lepton candidates $(\mathrm{e}, \mu$, or $\tau_{h}$ ) to pass the final identification and isolation criteria are measured in independent, highly pure control samples of multijet, $\mathrm{W} \rightarrow \mu v+$ jet, and $\mathrm{Z} \rightarrow \mu \mu+$ jet events. The control regions are exclusive to the signal region due to different final state topology, inverted isolation requirements, or both. To minimize possible biases, the same trigger, kinematic, and quality criteria used in the final analysis are applied to the control samples. Sidebands are defined for each channel, where the final identification or isolation criterion is not satisfied for one or more of the final-state lepton candidates. The sidebands are dominated by the non-prompt backgrounds. The number of non-prompt background events due to a lepton candidate being misidentified in the final selection is estimated by weighting each observed non-prompt lepton candidate in the sideband by its corrected probability $f\left(p_{\mathrm{T}}\right) /\left(1-f\left(p_{\mathrm{T}}\right)\right)$ to pass the final identification and isolation criteria. The background from $\ell^{ \pm} \ell^{\mp}$ events with a fake $\tau_{h}$ and where the charge of one of the light leptons is reconstructed incorrectly is negligible. In the $\ell \ell L L$ channels, at least one of the Higgs boson candidate leptons is misidentified in all non-prompt backgrounds, so the total fake estimate is the sum of the estimates from both. The sum of the two estimates from two leptons counts backgrounds where both leptons are non-prompt $(Z+2$ jets, multijet, etc $)$ twice. This double-counting is removed by inverting the identification of both leptons and applying both misidentification weights simultaneously, giving an independent estimate of events with two non-prompt leptons. The estimate
Table 1. Observed events and expected yields from the different background processes for the three and four-lepton channels.

The uncertainties represent the combined statistical and systematic uncertainties.

\begin{tabular}{l|c|c} 
Process & $\ell \ell \tau_{h}$ & $\ell \ell L L$ \\
\hline Fakes & $17.6 \pm 2.9$ & $16.9 \pm 6.4$ \\
$\mathrm{WZ}$ & $24.7 \pm 2.7$ & \\
\hline $\mathrm{ZZ}$ & $1.8 \pm 0.2$ & $19.0 \pm 2.6$ \\
\hline \hline Total bkg. & $44.0 \pm 4.1$ & $36.0 \pm 6.9$ \\
\hline $\mathrm{VH} \rightarrow \mathrm{V} \tau \tau\left(m_{H}=125 \mathrm{GeV}\right)$ & $2.6 \pm 0.3$ & $1.6 \pm 0.1$ \\
$\mathrm{VH} \rightarrow \mathrm{VWW}\left(m_{H}=125 \mathrm{GeV}\right)$ & $0.35 \pm 0.04$ & $0.74 \pm 0.06$ \\
\hline Observed & 46 & 45
\end{tabular}
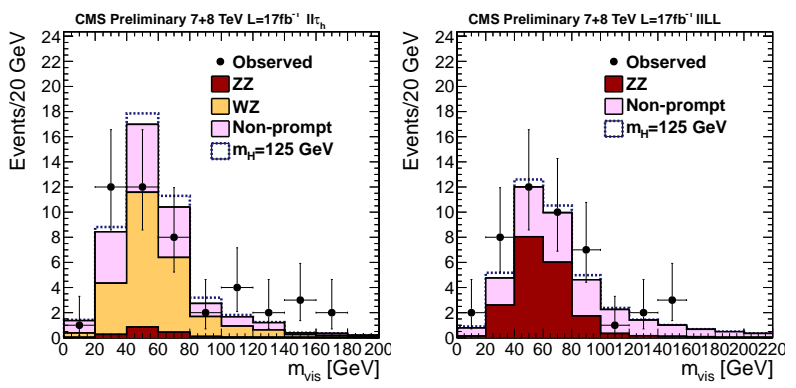

Figure 1. Observed and expected Higgs boson candidate mass spectra in the $\ell \ell \tau_{h}$ (left) and $\ell \ell L L$ (right) channels. The expected contribution from the associated production of a SM Higgs boson with mass $m_{H}=125 \mathrm{GeV}$ is shown by the dashed line. The yields of each process are determined using the same maximum likelihood fit to a signal-plus-background hypothesis used in the limit setting procedure.

of the double-counted background is subtracted from the total sum.

The irreducible diboson backgrounds are $\mathrm{WZ}$ and $\mathrm{ZZ}$ events in the $\ell \ell \tau_{h}$ channels, and $\mathrm{ZZ}$ events in the $\ell \ell L L$ channels are estimated using simulation. In the $\ell \ell \tau_{h}$ channel the irreducible WZ background is estimated using MC simulation. The $\mathrm{ZZ}$ background is largely reduced by the veto of events containing an additional e, $\mu$, or $\tau_{h}$ candidate, and is estimated using MC simulation. In the $\ell \ell L L$ channels, WZ events have at least one non-prompt lepton and are estimated using the misidentification probabilities described above. The dominant background comes from irreducible ZZ events, which are estimated using MC simulation.

\section{Results}

The numbers of events in data and expected yields from all backgrounds are enumerated in Table 1 . The observed data are compatible with the background expectation.

The primary observables used in this analysis are the visible invariant mass of the tau pair associated to the Higgs boson candidate. In the $\ell \ell \tau_{h}$ channels, it is not possible to definitively assign the same-charge electrons or 
muons to either the $\mathrm{W}$ or the Higgs boson candidate. However, as the signal is expected to be dominated by $\mathrm{H} \rightarrow \tau \tau$ decays, the final-state light leptons produced in the decays of the $\tau$ leptons have a softer $p_{\mathrm{T}}$ spectrum than light leptons from $\mathrm{W} \rightarrow l v$ decays, as they are associated with two neutrinos instead of one. Accordingly, we define the subleading light lepton and $\tau_{h}$ as the Higgs boson candidate. The observed and expected Higgs boson candidate visible mass spectra are shown in Fig. 1. The yields of each process are determined using the same maximum likelihood fit to a signal-plus-background hypothesis used in the limit setting procedure.

\section{Systematic Uncertainties}

The efficiencies for the Higgs boson signal and some of the background samples are estimated using MC simulation. The uncertainties on the simulation-to-data correction factors are taken as systematic uncertainties, and are propagated to the final results. The trigger, identification, and isolation efficiencies for electrons and muons are measured with data using the "tag-and-probe" technique [6] in $\mathrm{Z} \rightarrow \ell \ell$ events. The $\tau_{h}$ identification efficiency is measured with an uncertainty of $8 \%$ (per $\tau_{h}$ candidate) using the tag-and-probe technique in $\mathrm{Z} \rightarrow \tau \tau \rightarrow \mu \tau_{h}$ events [7]. Uncertainties on the jet energy scale (JES) have been evaluated in $\mathrm{Z}+$ jet and $\gamma+$ jet events [8] and vary between 2 and $5 \%$. The effect on the signal yields due to JES is approximately $1 \%$. There is a $2.2 \%$ uncertainty on the total integrated luminosity of the collected $7 \mathrm{TeV}$ data sample, and $4.4 \%$ on the $8 \mathrm{TeV}$ data sample. Two theoretical systematic uncertainties due to uncertainty on the QCD factorization and renormalization scale are considered.

For the non-prompt background estimation, the main systematic uncertainties are due to limited number of events in the weighted control region and uncertainty due to the misidentification rate which are between $15-35 \%$ depending on the final state. For the $\ell \ell \tau_{h}$ channels, the associated uncertainty on the WZ diboson backgrounds is $12 \%$ and is taken from the 2011 CMS cross section measurements. For the $\ell \ell L L$ channels, the theoretical uncertainty
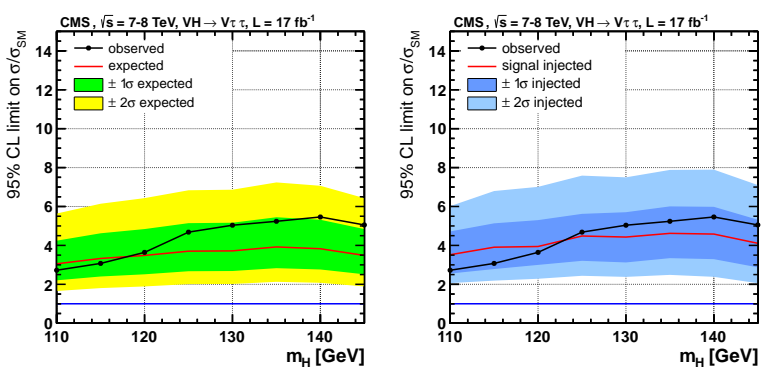

Figure 2. The expected and observed $95 \% \mathrm{CL}$ upper limits on SM Higgs boson production set by the combination of the analyses presented in this document are shown at left. At right, the observed limit is compared to the distribution of observed limits computed using many signal-injected pseudo-datasets. of $10 \%$ on the $\mathrm{ZZ}$ production cross section dominates the uncertainty on the estimate of the $\mathrm{ZZ}$ background.

\section{Limits on Higgs boson production}

The data show no evidence for the presence of a Higgs boson signal, and we set 95\% CL upper bounds on the Higgs boson cross section. To obtain exclusion limits we use the asymptotic $\mathrm{CL}_{s}$ method [9], based on a binned likelihood of the Higgs boson candidate visible invariant mass spectrum. The observed and median expected 95\% CL upper limits on SM Higgs boson production set by the combined analyses, are shown in Figs 2. The observed upper limits are also compared to the median observed limit expected in the presence of a SM Higgs boson, using a Monte Carlo "signal injection" technique. The observed limits set by this analysis are found to be statistically compatible with both the absence and presence of a SM Higgs boson.

\section{Summary}

An extended search for the associated production of standard model Higgs bosons decaying to tau pairs at the CMS experiment has been described. The search is conducted using events with three or four isolated leptons in $17 \mathrm{fb}^{-1}$ of 7 and $8 \mathrm{TeV}$ CMS data. The data are compatible with both the background-only prediction and the presence of a SM Higgs boson. Upper limits of 2.7 to 5.5 times greater than the predicted value are set at $95 \% \mathrm{CL}$ for the product of the SM Higgs boson production cross section and decay branching fraction in the mass range $110<m_{\mathrm{H}}<145$ GeV.

\section{References}

[1] CMS Collaboration, Phys. Lett. B 716, 30-61 (2012)

[2] ATLAS Collaboration, Phys. Lett. BB716, 1-29 (2012)

[3] CMS Collaboration, CMS-PAS-HIG-12-018 (2012)

[4] CMS Collaboration, CMS-PAS-HIG-12-051 (2012)

[5] CMS Collaboration, JINST 03, S08004 (2008)

[6] CMS Collaboration, JHEP 01, 080 (2011)

[7] CMS Collaboration, JINST 7, 1001 (2012)

[8] CMS Collaboration, JINST 6, 11002 (2012)

[9] Cowan, G et al. EPJC 71, 1554 (2011) 